quant-ph/0209032

\title{
Comment on "On the uncertainty relations and squeezed states for the quantum mechanics on a circle"
}

\author{
D.A. Trifonov \\ Institute for nuclear research, \\ 72 Tzarigradsko chaussee, 1784 Sofia, Bulgaria
}

November 10, 2018

\begin{abstract}
It is shown by examples that the position uncertainty on a circle, proposed recently by Kowalski and Rembieliński [J. Phys. A 35 (2002) 1405] is not consistent with the state localization. We argue that the relevant uncertainties and uncertainty relations (UR's) on a circle are that based on the Gram-Robertson matrix. Several of these generalized UR's are displayed and related criterions for squeezed states are discussed.
\end{abstract}

1. In the recent paper [1] the problem of relevant uncertainty relation (UR) for the angular momentum and the angle variables of a particle on a circle was discussed and a new UR was proposed. Noting a contradiction in the previously obtained UR [2] the authors defined new quantities $\tilde{\Delta}^{2}(\hat{\varphi})$ and $\tilde{\Delta}^{2}(\hat{J})(\hat{\varphi}=\varphi, \hat{J}=-i d / d \varphi)$ as measures for the uncertainty of the angle $\varphi$ and the angular momentum $J$ and suggest the inequality

$$
\tilde{\Delta}^{2}(\hat{\varphi})+\tilde{\Delta}^{2}(\hat{J}) \geq 1
$$

The quantities $\tilde{\Delta}^{2}(\hat{\varphi}), \tilde{\Delta}^{2}(\hat{J})$ are defined as [1] (note a change in notation: $\Delta^{2} \rightarrow \tilde{\Delta}^{2}$ )

$$
\tilde{\Delta}^{2}(\hat{\varphi})=-\frac{1}{4} \ln \left|\left\langle U^{2}\right\rangle\right|^{2}, \quad \tilde{\Delta}^{2}(\hat{J})=\frac{1}{4} \ln \left(\left\langle e^{-2 \hat{J}}\right\rangle\left\langle e^{2 \hat{J}}\right\rangle\right),
$$

where $U=\exp (i \hat{\varphi})$. The authors of [1] find that for the eigenstates $|z\rangle$ of the operator $Z=\exp (-\hat{J}+$ $1 / 2) U, Z|z\rangle=z|z\rangle$ (the "genuine coherent states (CS's) for a quantum particle on a circle" 3n) both quantities (2) equal $1 / 2$, and suggest that $\tilde{\Delta}^{2}(\hat{\varphi})$ and $\tilde{\Delta}^{2}(\hat{J})$ obey the inequality (11) in any state. Henceforth, the quantities $\tilde{\Delta}^{2}(\hat{\varphi})$ and $\tilde{\Delta}^{2}(\hat{J})$ should be referred to as Kowalski-Rembieliński uncertainties ((K-R) uncertainties), the UR (1) - as K-R UR and $|z\rangle$ - as Kowalski-RembielińskiPapaloucas CS's (K-R-P CS's).

2. Next we shall demonstrate that the K-R uncertainty $\tilde{\Delta}^{2}(\hat{\varphi})$ is not consistent with the state localization on a circle. For this purpose we compare the $\varphi$-probability distributions $p_{\psi}(\varphi)$ (defined as $\left.p_{\psi}(\varphi)=|\psi(\varphi)|^{2}=|\langle\varphi \mid \psi\rangle|^{2}\right)$ in K-R-P CS's with $\varphi$-distributions in certain states with squeezed $\tilde{\Delta}^{2}(\hat{\varphi})$. The quantity $\tilde{\Delta}^{2}(\hat{\varphi})$ is called [1] squeezed if it is less that than $1 / 2$. The authors of [1] constructed a family of such squeezed states $|z\rangle_{s}$ as eigenstates of the operator $Z(s)=\exp (-s \hat{J}+s / 2) U=$ $\exp (i \varphi-s \hat{J})$, where $s$ is positive parameter. Here we shall consider $\tilde{\Delta}^{2}(\hat{\varphi})$-squeezed states of the form of eigenstates $|z, a\rangle$ of the squared operator $Z^{2}$. These are defined as macroscopic superpositions of $|z\rangle$ and $|-z\rangle$ (Schrödinger cat states on a circle),

$$
|z, a\rangle=N(z, a)(|z\rangle+a|-z\rangle)
$$


where $a$ is complex parameter, and the normalization constant $N(z, a)$ takes the form

$$
N(z, a)=\left[1+|a|^{2}+2\langle z \mid-z\rangle \operatorname{Re} a\right]^{-1 / 2} .
$$

The scalar product of two CS's is [1] $\langle z \mid \eta\rangle=\theta_{3}\left((i / 2 \pi) \ln \left(z^{*} \eta\right), i / \pi\right)$, where $\theta_{3}(x, y)$ is the Jacobi theta-function. The states $|z, a= \pm 1\rangle \equiv|z ; \pm\rangle$ should be called even/odd CS on a circle.

On the states $|z, a\rangle$ the quantities $\left\langle U^{2}\right\rangle,\langle\exp (2 J)\rangle,\langle\exp (-2 J)\rangle$ in (2) take the form

$$
\begin{aligned}
\left\langle a, z\left|U^{2}\right| z, a\right\rangle & =N^{2}(z, a)\left(\left\langle z\left|U^{2}\right| z\right\rangle+|a|^{2}\left\langle-z\left|U^{2}\right|-z\right\rangle+a\left\langle z\left|U^{2}\right|-z\right\rangle+a^{*}\left\langle-z\left|U^{2}\right| z\right\rangle\right), \\
\left\langle a, z\left|e^{ \pm 2 J}\right| z, a\right\rangle & =N^{2}(z, a)\left(\left\langle z\left|e^{ \pm 2 J}\right| z\right\rangle+|a|^{2}\left\langle-z\left|e^{ \pm 2 J}\right|-z\right\rangle+2 \operatorname{Re}\left(a\left\langle z\left|e^{ \pm 2 J}\right|-z\right\rangle\right)\right),
\end{aligned}
$$

where $\left\langle z\left|U^{2}\right| z\right\rangle,\left\langle z\left|e^{2 J}\right| z\right\rangle$ and $\left\langle z\left|e^{-2 J}\right| z\right\rangle$ are given by $z / e z^{*}, e /|z|^{2}$ and $e|z|^{2}$ respectively [1]. Substituting (5) and (6) in (2) we obtain explicit formulas for $\mathrm{K}-\mathrm{R}$ uncertainties in $|z, a\rangle$.

From formulas (2), (5) and (6) we find that $\tilde{\Delta}^{2}(\hat{\varphi})$-squeezing occurs in many superpositions $|z, a\rangle$, in particular in $|z ; \pm\rangle$ (see figure 1). In the odd state $|1 ;-\rangle$, corresponding to the solid line minimum in figure 1 , we find $\widetilde{\Delta}^{2}(\hat{\varphi}) \approx 0.33$, which is considerably less than the value $1 / 2$ of $\tilde{\Delta}^{2}(\hat{\varphi})$ in CS's $|z\rangle$. One should expect that the $\varphi$-distribution, corresponding to wave functions with squeezed "position uncertainty" $\tilde{\Delta}^{2}(\hat{\varphi})$ are better localized on the circle than the non-squeezed CS. Unfortunately it is not the case with $\tilde{\Delta}^{2}(\hat{\varphi})$-squeezed states from the family $\{|z, a\rangle\}$. This inconsistency is demonstrated in figure 2 on the example of cat state $|1 ;-\rangle$. As one can see from figure 2 the $\tilde{\Delta}^{2}(\hat{\varphi})$-squeezed state $|1 ;-\rangle$ is much worse localized than the non-squeezed CS $|z\rangle(p(\varphi)$-distributions of $|z\rangle$ with different $z$ approximately coincide up to a translation). Therefore the quantity $\tilde{\Delta}^{2}(\hat{\varphi})$ is not a proper measure of the position uncertainty, and the inequality (1) could hardly be qualified as a relevant uncertainty relation on a circle.

Let us note that $|z, a\rangle$ saturate the inequality (11) with unequal $\tilde{\Delta}^{2}(\hat{\varphi})$ and $\tilde{\Delta}^{2}(\hat{J})$, the case of $z=1$ and real $a$ being demonstrated in figure 1 . However the whole range of validity of (1) is not yet clarified. Nevertheless it might be interesting to note that in the variety of states on the real line a similar inequality hold, i.e. $\tilde{\Delta}^{2}(\hat{x})+\tilde{\Delta}^{2}(\hat{p}) \geq 1$, where $\hat{x}, \hat{p}$ are position and momentum operators.

3. The above remarks naturally raise again the questions about the position and angular momentum uncertainties and the relevant uncertainty relations (UR's) on a circle. In my opinion most suitable UR's for $n$ observables $X_{i}$ and one state $|\psi\rangle$ on a circle are those based on the Gram-Robertson matrix $G=\left\{G_{i j}\right\}$ of the form 四 $(i, j=1, \ldots, n ; n=1,2, \ldots)$

$$
G_{i j}(\psi)=\left\langle\left(X_{i}-\left\langle X_{i}\right\rangle\right) \psi \mid\left(X_{j}-\left\langle X_{j}\right\rangle\right) \psi\right\rangle .
$$

More informative notations $G(\vec{X} ; \psi)$ and $G_{X_{i} X_{j}}(\psi)\left(\vec{X}=X_{1}, X_{2}\right)$ for this matrix and its elements should also be used. The generalized covariances ${ }_{g} \Delta X_{i} X_{j}(\psi)$ of $X_{i}$ and $X_{j}$ in $|\psi\rangle$ are defined [4] as symmetric part $S_{i j}$ of $G_{i j}$ (for the case of $n=2$ see also [5, 6])

$$
{ }_{g} \Delta X_{i} X_{j}(\psi):=S_{X_{i} X_{j}}(\psi)=\operatorname{Re}\left\langle\left(X_{i}-\left\langle X_{i}\right\rangle\right) \psi \mid\left(X_{i}-\left\langle X_{i}\right\rangle\right) \psi\right\rangle .
$$

The diagonal elements $S_{i i}$ are defined as generalized variances $\left({ }_{g} \Delta X_{i}\right)^{2}$ of $\left.X_{i}\right)$.

Since $G$ is non-negative all the characteristic coefficients of its symmetric part $S=\left(G+G^{T}\right) / 2$ are not less than the corresponding characteristic coefficients of its antisymmetric part $A=\left(G-G^{T}\right) / 2 i$. These inequalities are called generalized characteristic UR's 团. The senior characteristic UR reads

$$
\operatorname{det} S(\vec{X} ; \psi) \geq \operatorname{det} A(\vec{X} ; \psi) \text {. }
$$

In the simplest case of $n=2$ this UR is displayed as $S_{11} S_{22}-S_{12}^{2} \geq A_{12}^{2}$. It can also be written in the shorter form $\operatorname{det} G \geq 0$, and displayed in terms of the generalized covariances as

$$
\left({ }_{g} \Delta X_{1}\right)^{2}\left({ }_{g} \Delta X_{2}\right)^{2} \geq\left({ }_{g} \Delta X_{1} X_{2}\right)^{2}+\left(\operatorname{Im}\left\langle\left(X_{1}-\left\langle X_{1}\right\rangle\right) \psi \mid\left(X_{2}-\left\langle X_{2}\right\rangle\right) \psi\right\rangle\right)^{2} .
$$

The sum of the two terms in the right-hand-side of (10) is just the squared absolute value of $G_{12}$, i.e. we have ${ }_{g} \Delta X_{1}{ }_{g} \Delta X_{2} \geq\left|G_{12}(\psi)\right|$. 
When the actions of $X_{i} X_{j}$ on $|\psi\rangle$ are correctly defined (normal cases) the above Gram matrix

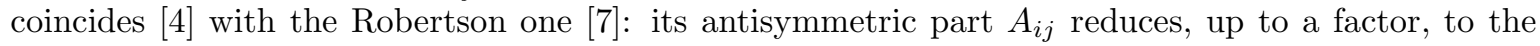
mean commutator, $A_{i j}=-(i / 2)\left\langle\left[X_{i}, X_{j}\right]\right\rangle$, and its symmetric part takes the familiar form of the standard uncertainty matrix $\sigma(\vec{X} ; \psi)$. (The element $\sigma_{i j}=\left\langle X_{i} X_{j}+X_{j} X_{i}\right\rangle / 2-\left\langle X_{i}\right\rangle\left\langle X_{j}\right\rangle \equiv \Delta X_{i} X_{j}$ is standard covariance of $X_{i}$ and $X_{j}$, and $\sigma_{i i}=\Delta X_{i} X_{i} \equiv\left(\Delta X_{i}\right)^{2}$ is the variance of $X_{i} .(\Delta X)^{2}$ should not be confused with the K-R quantity $\left.\tilde{\Delta}^{2}(X)\right)$. Under these conditions the inequality (9) takes the form of Robertson UR for $n$ observables [7, 4 , 8], and (10) coincides with the Schrödinger (or Schrödinger-Robertson) UR [9] (for a review on this UR and its minimization states see e.g. [8]).

The generalized form of the less precise Heisenberg UR reads ${ }_{g} \Delta X_{1}{ }_{g} \Delta X_{2} \geq\left|\operatorname{Im} G_{12}\right|$, and it again follows from the more precise one (10). About similar generalization see also $\sqrt{5}$, 6 . (Please note that in some papers, e.g. [10], no distinction is made between Schrödinger and Heisenberg UR's, both being named after Heisenberg).

Thus in the special cases when $X_{i} X_{j}|\psi\rangle$ are not properly defined one should resort to generalized Schrödinger UR (10) (for two observables), and to (9) (for several observables). The position and the angular momentum observables of a particle on a circle represent such a special case, since $\hat{\varphi}\langle\varphi \mid \psi\rangle=$ $\varphi\langle\varphi \mid \psi\rangle$ is not $2 \pi$-periodic and $\hat{J}$ is not Hermitian on such functions. Another special case of interest is particle motion on the sphere.

Figure 3 illustrates the generalized UR (10) in the case of $X_{1}=\hat{J}$ and $X_{2}=\hat{\varphi}$ and states $|z, a\rangle$ (particle on a circle), where $\operatorname{det} G(z, a)=\operatorname{det} G(\vec{X} ; z, a)$ are plotted as functions of real $a$ for $z=0.4$ (solid line) and $z=1$ (dashed line). In these states the generalized covariance ${ }_{g} \Delta \varphi J=\operatorname{Re} G_{J \varphi}$ vanishes, also ${ }_{g} \Delta \varphi=\Delta \varphi,{ }_{g} \Delta J=\Delta J$, so that here we have $\operatorname{det} G=(\Delta \varphi)^{2}(\Delta J)^{2}-\left(\operatorname{Im} G_{J \varphi}\right)^{2} \geq 0$. The minimal value of $\operatorname{det} G$ on figure 3 is different from zero (it is about 0.00017 ).

Unlike $\tilde{\Delta}^{2}(\hat{J})$ and $\tilde{\Delta}^{2}(\hat{\varphi})$, the variances $(\Delta J)^{2}$ and $(\Delta \varphi)^{2}$ are in good correspondence with the angular momentum and position localization on a circle. For example, $\varphi$-distributions for CS's $|z\rangle$ with $z=0.4,1$ practically are the same (see figure 2), and the variances $(\Delta \varphi)^{2}$ in these CS are almost equal: in $|z=0.4\rangle(\Delta \varphi)^{2}=0.50055$, and in $|z=1\rangle(\Delta \varphi)^{2}=0.50064$. In the worse localized cat state $|1 ;-\rangle$ (see figure 2 ) the variance $(\Delta \varphi)^{2}$ takes the larger value of 3.813 .

We have to warn that one has to be careful about the correspondence between $\Delta \varphi$-squeezing and localization of the wave function $\langle\varphi \mid \psi\rangle$ : in view of the identification of points $\varphi$ and $\varphi+2 \pi$ the meanvalues $\langle\varphi\rangle,\left\langle\varphi^{2}\right\rangle$ should be calculated by integration from $\varphi_{0}-\pi$ to $\varphi_{0}+\pi$, where $\varphi_{0}$ is the center of the wave packet (i.e. $\varphi_{0}$ is the most probable value of $\varphi$ ). In this way we find that both standard deviations $\Delta \varphi$ and $\Delta J$ in K-R-P CS's $|z\rangle$ show very small oscillations around the value of $1 / 2$. So, the family $\{|z\rangle\}$ consists of almost minimum uncertainty states on the circle.

4. The minimization states (intelligent, or minimum-uncertainty states) of the generalized UR (10) for $X_{1}$ and $X_{2}$ should be eigenstates of a real or complex combination $\mu X_{1}+\nu X_{2}$. In the case of the particle on a circle and $X_{1}=\hat{J}$ and $X_{2}=\hat{\varphi}$ the $2 \pi$-periodicity condition on the wave functions $\psi(\varphi+2 \pi)=\psi(\varphi)$ should be imposed (some authors admit exceptions [6]). This restriction rules out all solutions of the eigenvalue equation $(\mu \hat{J}+\nu \hat{\varphi})|\psi\rangle=z|\psi\rangle$, except for the eigenstates $\psi_{m}(\varphi)$ of $\hat{J}$, $\psi_{m}(\varphi)=(1 / \sqrt{2 \pi}) \exp (i m \varphi)$. For $\psi_{m}(\varphi)$ we have $\Delta J=0, \Delta \varphi=\sqrt{\pi}, G_{J \varphi}=0$, so that the equality in UR (10) reads $0=0$. None of the states $|z, a\rangle$ and $|z\rangle_{s}$ minimize the inequalities (10), although the deviations in the case of CS's $|z\rangle$ are very small, as it is illustrated in figure 3 at $a=0$.

In order to define squeezed states on the circle let us recall that for the particle on the real line these states are defined by means of one of the two inequalities $(\Delta x)^{2}<|\langle[x, p]\rangle| / 2=1 / 2$, or $(\Delta p)^{2}<$ $|\langle[x, p]\rangle| / 2=1 / 2$. Since $\operatorname{Im} G_{12}(\psi)$ is a generalization of the mean commutator $(-i / 2)\left\langle\left[X_{1}, X_{2}\right]\right\rangle$ one can define $X_{1}-X_{2}$ squeezed states more generally as states, for which

$$
\left({ }_{g} \Delta X_{i}\right)^{2}<\left|\operatorname{Im} G_{12}(\psi)\right|, \quad i=1 \text { or } 2 .
$$

This is a generalization of the well known Eberly-Wodkiewicz criterion for squeezed states. It however is a relative criterion, since the "generalized mean commutator" $\left|\operatorname{Im} G_{12}(\psi)\right|$ may take, in general, values from 0 to $\infty$. Another and stronger criterion for squeezed states is suggested by the observation that on the real line (and for the one mode electromagnetic field) $1 / 2$ is the minimal value that two variances $(\Delta x)^{2}$ and $(\Delta p)^{2}$ can take simultaneously. Therefore we can define $X_{1}-X_{2}$ squeezed states 
more generally as states, for which one of the following two inequalities holds,

$$
\left({ }_{g} \Delta X_{i}\right)^{2}<\Delta_{0}^{2}, \quad i=1 \text { or } 2,
$$

where $\Delta_{0}^{2}$ is the minimal value that the two generalized variances can take simultaneously. For incompatible observables $\Delta_{0}>0$. It is plausible that $2 \Delta_{0}^{2}$ is the lower limit of the sum of two variances,

$$
\left(\Delta X_{1}\right)^{2}+\left(\Delta X_{2}\right)^{2} \geq 2 \Delta_{0}^{2}
$$

If the eigenstates of $X_{1}+i X_{2}$ (or $X_{1}-i X_{2}$ ) exist (canonical observables, spin and quasi-spin components e.t.c.), then $\Delta_{0}^{2}$ is equal to the minimal value of $\left|\operatorname{Im} G_{J \varphi}(\psi)\right|$ within these eigenstates, and (13) is rigorously valid [8]. If eigenstates of $X_{1} \pm i X_{2}$ do not exist, the critical quantity $\Delta_{0}$ should be estimated by different methods. The case of $X_{1}=\hat{J}$ and $X_{2}=\hat{\varphi}$ is such a special case, since $2 \pi$-periodic eigenfunctions of $\hat{\varphi} \pm i \hat{J}$ do not exist. Numerical considerations suggest that in this case $\Delta_{0}^{2} \approx 0.5$ (more precisely $\approx 0.49999$ ), which is the minimal value that $(\Delta \varphi)^{2}$ and $(\Delta J)^{2}$ take simultaneously in CS's $|z\rangle$.

It turned out that both criterions (11) and (12) can be satisfied in many states from the families $\{|z, a\rangle\}$ and $\left\{|z\rangle_{s}\right\}$. Squeezing of $\Delta \varphi$ in $|z, a\rangle$ is not very strong, while in $|z\rangle_{s}$ it can be arbitrarily strong.

Of course $|z\rangle$ are exact Heisenberg intelligent states for the Hermitian components $X, Y$ of $Z$. However neither $\Delta X$ nor $\Delta Y$ is in a satisfactory correspondence with the localization on a circle, as one can easily check it on the example of cat states $|z ; \pm\rangle$.

In conclusion we note that the above described scheme can be extended to represent correct generalized UR's for several observables and (several) mixed states as well 顿.

\section{References}

[1] Kowalski K. and Rembieliński J. 2002, On the uncertainty relations and squeezed states for the quantum mechanics on a circle, J. Phys. A: Math. Gen. 35 1405-1414 quant-ph/0202070.

[2] Gonzáles J.A. and del Olmo M.A. 1998, Coherent states on the circle, J. Phys. A 318841.

[3] Kowalski K., Rembieliński J. and Papaloucas L.C. 1996, Quantum mechanics on a sphere and coherent states, J. Phys. A: Math. Gen. 294149.

[4] Trifonov D.A. 2000, State extended uncertainty relations, J. Phys. A: Math. Gen. 33 L299 quantph/0005086; Trifonov D.A. 2001, Remarks on the extended characteristic uncertainty relations, J. Phys. A: Math. Gen. 34 L75.

[5] E.D. Chisolm, Generalizing the Heisenberg uncertainty relation, Am. J. Phys. 69, 368-371 (2001) quant-ph/0011115.

[6] S. Dumitru, On a subject of diverse improvisations: The uncertainty relations on a circle, E-print quant-ph/0206009.

[7] H.P. Robertson, An indeterminacy relation for several observables and its classical interpretation, Phys. Rev. 46, 794 (1934).

[8] D.A. Trifonov, Schrödinger uncertainty relation and its minimization states, World of Phys. 24 (2001) 107 physics/0105035]; Generalized uncertainty relations and coherent and squeezed states, JOSA A 17, 2486 (2000).

[9] E. Schrödinger, Zum Heisenbergschen Unschärfeprinzip, Sitz. Preus. Acad. Wiss. (Phys.-Math. Klasse), 19, 296-303 (1930); H. P. Robertson, A general formulation of the uncertainty principle and its classical interpretation, Phys. Rev. 35, 667-667 (1930) (abstract only).

[10] G.S. Agarwal, Heisenberg's uncertainty relations and quantum optics, Fortschritte der Physik 50, 575 (2002) quant-ph/0201098. 


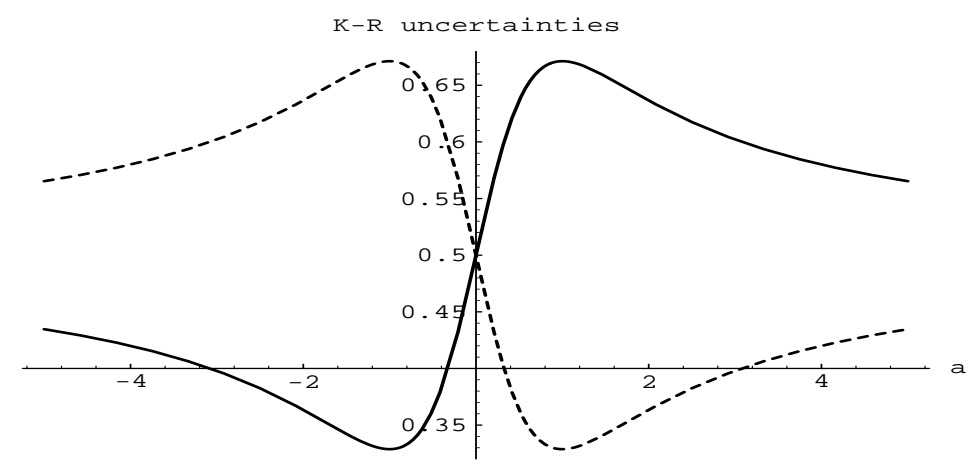

Figure 1: K-R uncertainties $\tilde{\Delta}^{2}(\hat{\varphi})$ (solid line) and $\tilde{\Delta}^{2}(\hat{J})$ (dashed line) in cat states $|z=1, a\rangle$ as functions of $a . \tilde{\Delta}^{2}(\hat{\varphi})$-squeezing is maximal around $a=-1$.

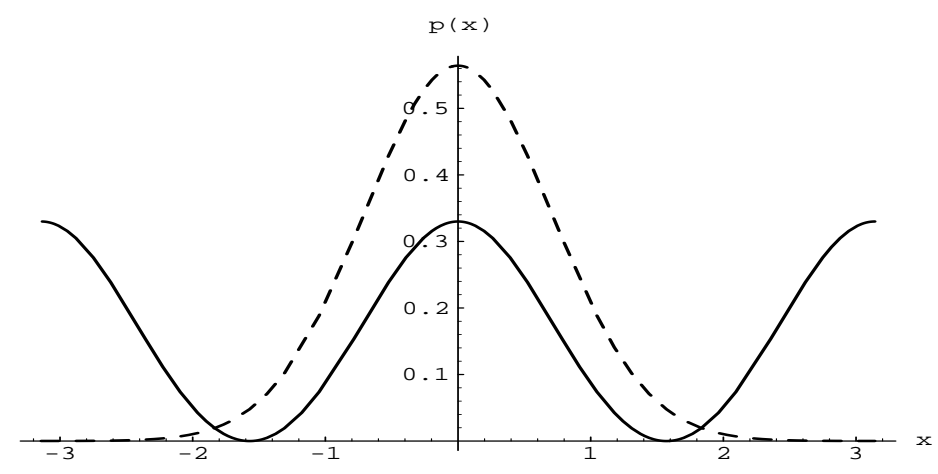

Figure 2: The distributions $p(x)$ as functions of the angle $\varphi \equiv x$ for $\tilde{\Delta}^{2}(\hat{\varphi})$-squeezed state $|1 ;-\rangle$ (solid line) and non-squeezed CS $|z=1\rangle$ (dashed line). CS $|1\rangle$ is better localized than $|1 ;-\rangle$.

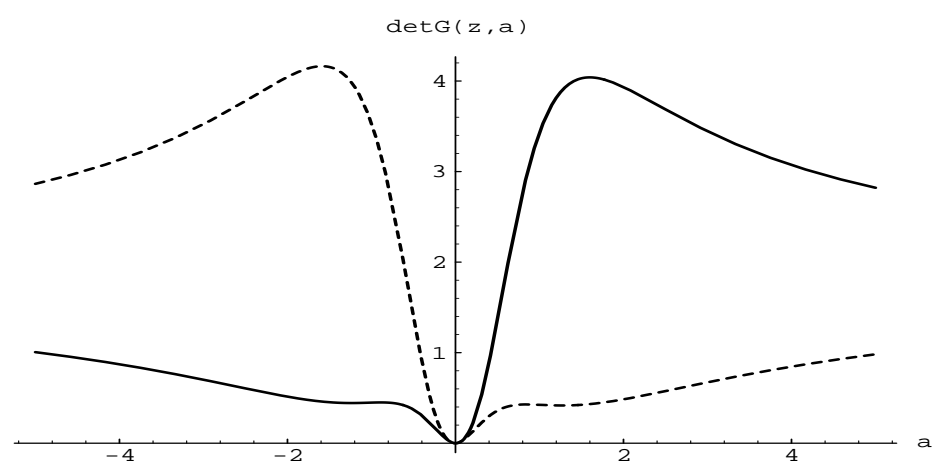

Figure 3: Illustration of the generalized Schrödinger uncertainty relation (10) in the superpositions $|z, a\rangle: \operatorname{det} G=(\Delta \varphi)^{2}(\Delta J)^{2}-\left|G_{J \varphi}\right|^{2}$ as a function of $a$ for $z=0.4$ (solid line) and $z=1$ (dashed line). 